\title{
EMBEDDING UP TO HOMOTOPY TYPE IN EUCLIDEAN SPACE
}

\author{
A.N. DRANišnikov AND D. REPOV̌̃
}

We give a short proof of the classical Stallings theorem that every finite $\boldsymbol{n}$ dimensional cellular complex embeds up to homotopy in the 2n-dimensional Euclidean space. As an application we solve a problem of $M$. Kreck.

\section{INTRODUCTION}

This note was inspired by a question of Kreck during his visit to the Steklov Mathematical Institute in Spring of 1989: Can every finitely presented group be realised as the fundamental group of a 2-dimensional polyhedron embedded in $\mathbb{R}^{4}$ and can such polyhedron have a minimal Euler characteristic?

We recall that not every 2-polyhedron is embeddable in 4-dimensional Euclidean space [4]. It turns out that the answer to the first question follows from the classical theorem of Stallings [9]:

Stallings Theorem. For every finite $n$-dimensional $(n>0)$ cellular complex $K$ there exists a polyhedron $M$, homotopy equivalent to $K$, which is embeddable in $\mathbb{R}^{2 n}$.

After having seen our solution of his problem in 1991, Kreck kindly informed us about the recent work of Huck [5], through which we became familiar with Stallings' mimeographed notes [9] and some other related papers $[1,2,3,8,11]$. Our solution of Kreck's problem provides an alternative (and we believe also simpler) proof of the Stallings theorem.

The final version of this paper was prepared during the visit by the second author to the Steklov Mathematical Institute in 1991, on the basis of the long term agreement between the Slovene Academy of Arts and Sciences and the Soviet Academy of Sciences (1991-1995).

Received 20 January 1992

Supported in part by a grant from the Ministry of Science and Technology of the Republic of Slovenia.

Copyright Clearance Centre, Inc. Serial-fee code: 0004-9729/93 \$A2.00+0.00. 


\section{Preliminaries}

Our proof is based on the following two lemmas:

LEMMA 1. Suppose that $f: X \rightarrow Y$ is a homotopy equivalence between cellular complexes and $g: S^{n} \rightarrow X$ is an attaching map for an $(n+1)$-dimensional cell $B^{n+1}$. Then $f$ can be extended to a homotopy equivalence $F: X \cup_{g} B^{n+1} \rightarrow Y \cup_{f \circ g} B^{n+1}$.

Proof: Standard argument - see for example in [10] or [6].

Lemma 2. Suppose that a polyhedron $P$ lies in $\mathbb{R}^{2 n} \times 0 \subset \mathbb{R}^{2 n} \times \mathbb{R}_{+}^{2}$ where $O$ is a point in the boundary of semi-plane $\mathbb{R}_{+}^{2}$ and let $f: S^{n} \rightarrow P$ be a map of the boundary of the $(n+1)$-dimensional ball. Then for every $\varepsilon>0$ there exists a map $g: B^{n+1} \rightarrow \mathbb{R}_{+}^{2 n+2}=\mathbb{R}^{2 n} \times \mathbb{R}_{+}^{2}$ such that

(1) the restriction $\left.g\right|_{\operatorname{Int} B^{n+1}}$ is an embedding in Int $\left(\mathbb{R}_{+}^{2 n+2}\right)$; and

(2) the restriction $\left.g\right|_{\partial B^{n+1}}=q$ is $\varepsilon$-close to $f$ and $\operatorname{Im}(q) \subset \mathbb{R}^{2 n} \times O$.

Proof: Choose $q: S^{n} \rightarrow \mathbb{R}^{2 n} \times O$ to be $\varepsilon$-close to $f$ and with general position properties. This means that there are only finitely many points of self-intersection of $q\left(S^{n}\right)$. Let $X \subset S^{n}$ be the singular set of $q$.

Fix an arbitrary point $v$ in $\mathbb{R}^{2 n} \times O$ from the $\varepsilon$-neighbourhood of $P$ and define a map $g_{1}: B^{n+1} \rightarrow \mathbb{R}^{2 n} \times O$ as the cone of map $q$ with vertex $v$. Therefore the map $g_{1}$ sends linearly the interval $[x, c]$ to the interval $[q(x), v]$ for each $x \in \partial B^{n+1}$, where $c$ is the centre of the ball $B^{n+1}$.

Let $O$ be the origin of some orthogonal coordinate system in $\mathbb{R}_{+}^{2}$ with the $x$-axis lying in the boundary and let $A, B$ and $C$ be the points with coordinates $(-1,1 / 2)$, $(1,1 / 2)$ and $(0,1)$, respectively. Since $X$ is 0 -dimensional one can choose a map $\phi: \partial B^{n+1} \rightarrow[A, B]$ on the interval $[A, B]$ such that $\phi \mid x$ is an embedding.

We define a map $g_{2}$ onto the boundary of a concentric ball of half the radius $(1 / 2) B^{n+1}$ as the composition $g_{2}=\phi \circ(\times 2)$. Here $(\times 2)$ sends $\partial\left((1 / 2) B^{n+1}\right)$ to $\partial B^{n+1}$ homeomorphically. Define $g_{2}\left(\partial B^{n+1}\right)=O$ and $g_{2}(c)=C$ and extend $g_{2}$ onto Int $(1 / 2) B^{n+1}$ and onto Int $B^{n+1}-(1 / 2) B^{n+1}$ linearly. Define $g=\left(g_{1}, g_{2}\right)$.

The properties (2) and (3) hold by the construction of $g$. Assume that $g(x)=g(y)$ for some $x, y \in \operatorname{Int} B^{n+1}$. We identify $B^{n+1}$ with the set $\left\{x \in \mathbb{R}^{n}:|x| \leqslant 1\right\}$. It is easy to see that $x \neq 0$ and $y \neq 0$. If $q(x /|x|)=q(y /|y|)$ then $x /|x|, y /|y| \in X$ and hence $g_{2}(x) \neq g_{2}(y)$. If $q(x /|x|) \neq q(y /|y|)$ then the equation $g_{1}(x)=g_{1}(y)$ implies that the points $q(x /|x|), q(y /|y|)$ and $c$ are collinear and therefore $|x| \neq|y|$. In that case $g_{2}(x) \neq g_{2}(y)$. Contradiction.

\section{Proof of Stallings theorem}

We shall use induction on $n$. Since every finite 1-dimensional complex is homotopy equivalent to a finite disjoint union of wedges of circles, the theorem is true for $n=1$. 
Let us now verify the inductive step. Let $K$ be an $(n+1)$-dimensional cellular complex and let $K^{(n)}$ denote the n-skeleton of $K$. By induction, there is a homotopy equivalence $f: K^{(n)} \rightarrow L$, where $L$ is a polyhedron embeddable in $\mathbf{R}^{2 n}$. Let $\left\{e_{i}: \partial B^{n+1} \rightarrow K^{(n)}\right\}_{i \leqslant m}$ be the family of attaching maps in $K$ for $(n+1)$-dimensional cells. Suppose that $\left\{\alpha_{i}\right\}_{i \leq m}$ is a family of angles on the plane with common vertex $O$ such that $\alpha_{i} \cap \alpha_{j}=O$ for $i \neq j$. Note that each $\alpha_{i}$ is homeomorphic to the halfplane $\mathbb{R}_{+}^{2}$. Let $N$ be a regular neighbourhood of $L$ in $\mathbb{R}^{2 n} \times 0$. There is $\delta>0$ such that the $\delta$-neighbourhood of $L$ is contained in $N$.

Apply Lemma 2 for $L, f \circ e_{i}$ and $\varepsilon=\delta$ to obtain maps $g_{i}: B^{n+1} \rightarrow \mathbb{R}^{2 n} \times \alpha_{i}$ with the properties (1) and (2). The property (2) implies that the map $g_{i}=g_{i} l_{\theta B^{n+1}}$ is homotopic to $f \circ e_{i}$ in $N$. The property (1) yields an embedding of

$$
M=N \underset{q 1}{\cup} B^{n+1} \cup_{q 2} B^{n+1} \cup \ldots \cup \underset{q m}{\cup} B^{n+1}
$$

in $\mathbb{R}^{2 n+2}$. Since we may assume that each map $q_{i}$ is simplicial with respect to some triangulations on $N$ and $\partial B^{n+1}$ we may regard $M$ as a polyhedron. Lemma 1 implies that $M$ is homotopy equivalent to $K$.

\section{Epilogue}

Note that by the construction $\operatorname{dim} M=2 \operatorname{dim} K-2$ and that we can also achieve that the Euler characteristics of $M$ be minimal. As a result we get the answer to both Kreck's questions:

Corollary. For every finitely presented group $G$ there exists a 2-dimensional polyhedron $M \subset \mathbb{R}^{4}$ with the fundamental group $\pi_{1}(M) \cong G$. In addition, we may assume that $M$ has minimal Euler characteristic.

REMARK. Another solution of Kreck's problem follows from the recent work of Skopenkov, Ščpin and the second author [7].

\section{REFERENCES}

[1] F. Connolly and B. Williams, 'Embeddings up to homotopy type and geometric suspensions of manifolds', Quart. J. Math. Oxford Ser. (2) 29 (1978), 385-401.

[2] G. Cooke, 'Embedding certain complexes up to homotopy type in Euclidean space', Ann. of Math. 90 (1969), 144-156.

[3] M.L. Curtis, 'On 2-complexes in 4-space', in Topology of 3-manifolds and related topics, Editor M.K. Fort Jr. (Prentice-Hall, Englewood Cliffs, N.J., 1962), pp. 204-207.

[4] A. Flores, 'Ūber $n$-dimensionale Komplexe, die im $R_{2 n+1}$ absolut selbstverschlungen sind', Ergeb. Math. Kolloq. 6 (1933/34), 4-7. 
[5] G. Huck, 'Embeddings of acyclic 2-complexes in $S^{4}$ with contractible complement', in Proc. Sem. Top. Comb. Group. Theory 1985-1988, Lecture. Notes Math. 1440, Editor P. Latiolais (Springer-Verlag, Berlin, Heidelberg, New York, 1990), pp. 122-129.

[6] M.M. Postnikov, Lectures on algebraic topology. Foundations of homotopy theory, (in Russian) (Nauka, Moscow, 1984).

[7] D. Repovš, A.B. Skopenkov and E.V. Šcepin, Embedding the mapping cylinder up to homotopy in Euclidean space (Steklov Math. Institute, Moscow, 1991). (preprint) .

[8] A. Shapiro, 'Obstructions to the embedding of a complex in a euclidean space. The first obstruction', Ann. of. Math. 66 (1957), 256-269.

[8] J. Stallings, The embedding of homotopy types into manifolds, mimeographed notes (Princeton University, Princeton, 1965).

[10] G.W. Whitehead, Elements of homotopy theory, Graduate Texts in Math 61 (SpringerVerlag, Berlin, Heidelberg, New York, 1978).

[11] W.-T. Wu, A theory of imbedding, immersion and isotopy of polytopes in euclidean space (Science Press, Peking, 1969).

Institute for Mathematics, Physics and Mechanics

University of Ljubljana

Ljubljana 61111

Slovenia 